\title{
Biot-Savart-like law in electrostatics
}

\author{
Mário H. Oliveira and José A.Miranda* \\ Laboratório de Física Teórica e Computacional, Departamento de Física, \\ Universidade Federal de Pernambuco, Recife, PE 50670-901 Brazil
}

(March 27, 2018)

\begin{abstract}
The Biot-Savart law is a well-known and powerful theoretical tool used to calculate magnetic fields due to currents in magnetostatics. We extend the range of applicability and the formal structure of the Biot-Savart law to electrostatics by deriving a Biot-Savart-like law suitable for calculating electric fields. We show that, under certain circumstances, the traditional Dirichlet problem can be mapped onto a much simpler Biot-Savart-like problem. We find an integral expression for the electric field due to an arbitrarily shaped, planar region kept at a fixed electric potential, in an otherwise grounded plane. As a by-product we present a very simple formula to compute the field produced in the plane defined by such a region. We illustrate the usefulness of our approach by calculating the electric field produced by planar regions of a few nontrivial shapes.
\end{abstract}

\footnotetext{
${ }^{*}$ Corresponding author

e-mail:jme@lftc.ufpe.br
} 


\section{INTRODUCTION}

The Biot-Savart law [1] is one of the most basic relations in electricity and magnetism. It allows one to determine the total magnetic field $\mathbf{B}$ at a given point in space as the superposition of infinitesimal contributions $\mathbf{d B}$, caused by the flow of current $I$ through an infinitesimal path segment $\mathbf{d s}$, oriented in the same direction as the current. If $\hat{\mathbf{r}}$ is the position unit-vector pointing from the element of length to the observation point $P$, then the total field contribution due to a closed circuit $C$ at $P$ is given by a closed line integral [1 14

$$
\mathbf{B}=\frac{\mu_{0} I}{4 \pi} \oint_{C} \frac{(\mathbf{d} \mathbf{s} \times \hat{\mathbf{r}})}{r^{2}}
$$

where $\mu_{0}$ is the permeability of free space and the path of integration is along the wire.

The usefulness of the Biot-Savart law goes far beyond its basic textbook applications. Equation (1) is a much studied integral form which arises in various interesting physical problems involving topological defects [5], magnetic fluids [6], amphiphilic monolayers [7], type-I superconductors [8] and the $n$-body problem of celestial mechanics [9]. In the framework of classical electrodynamics the Biot-Savart law provides a useful technique to calculate the magnetic field generated by current carrying wires. Equation (11) is quite general, valid for nonplanar current loops of arbitrary shape. Obviously, the presence of a cross-product in the formula may introduce some difficulties in practical calculations. Ultimately, the range of problems to which equation (11) can be applied is limited primarily by the difficulty experienced in performing the integrations. These difficulties are less serious if the loop $C$ is flat and if the observation point lies in the plane of the loop. It has been recently shown 10 that the magnitude of the magnetic field due to an arbitrarily shaped (not self-intersecting), planar, current carrying wire at a point lying on the wire's plane can be written as

$$
B=\frac{\mu_{0} I}{4 \pi} \oint_{C} \frac{d \theta}{r}
$$


Equation (2) is very simple and compact, expressing $B$ in terms of the wire shape $r=r(\theta)$, where $\theta$ denotes the polar angle. As shown in reference [10], this basic result expands the degree of applicability of the Biot-Savart law allowing exact, closed form solutions for a whole new set of elementary problems.

Based on the general nature of the Biot-Savart law, its connection to recent physics research topics [6] 9], and its success in performing magnetic field calcultions, we felt motivated to investigate the following question: is it possible to formulate a Biot-Savart-like law in electrostatics? If so, what sort of electrostatic problem could be more easily solved by such an approach? In this work we address these issues and show, for the first time, that it is indeed possible to formulate a Biot-Savart-type law in the realm of electrostatics.

The formulation we propose is suitable to calculate the electric field due to an arbitrary shaped, planar region maintained at a fixed scalar potencial $\mathrm{V}$, with the rest of the plane held at zero potential. We show that the calculation of the electric field produced by such a region is analogous to the evaluation of the magnetic field due to a flat current carrying wire of the same shape (figure 1). We point out that the "brute-force" calculation of the electric field due to such an arbitrarily shaped region, kept at a fixed potential, by directly applying conventional boundary-value techniques [11] may be quite challenging. The nontrivial nature of the problem comes from the fact that we do not know the surface charge distribution in advance. Our eletrostatic Biot-Savart law provides a much simpler way to perform this nontrivial electric field calculation, and allows one to borrow many of the standard procedures ordinarily used in the corresponding magnetostatic situation. Therefore, complicated electrostatic problems may have straightforward solutions, if solved by the Biot-Savart-like approach we develop here. We stress that, even though our derivation and new results (8) and (9) presented below are quite simple, they have not been derived in any standard electromagnetism book or journal publication. This work comes to fill this gap, offering a new 
and simple tool to perform electric field calculations.

As a by-product, we obtain a very compact expression for the electric field in the plane of the flat sheet (see equation (9)), which is as simple as its magnetic field counterpart given by equation (2). Finally, we illustrate our results by explicitly calculating the electric field produced by a constant potential, flat region that has the shape of a regular, $n$-sided polygon, for observation points located along its axis of symmetry. The calculation for the field produced in the plane of regions having other peculiar shapes (elliptical, spiral, regularly undulating) is also presented.

\section{ELECTRIC FIELD CALCULATION}

Consider an arbitrarily shaped, two-dimensional region, located in the $x-y$ plane, kept at a fixed potential $\mathrm{V}$ while the rest of the plane is held at zero potential (see figure 2). The shape of the flat plate is determined by a closed boundary curve $C$. We want to calculate the electric field caused by this charge configuration at a given observation point $\mathrm{P}$ located by the vector $\mathbf{x}$

$$
\mathbf{E}(\mathbf{x})=-\nabla \Phi(\mathbf{x})
$$

where $\Phi(\mathbf{x})$ denotes the electric scalar potential.

Since the scalar potential is specified everywhere on the $x-y$ plane, we have to solve a Dirichlet problem [11]. We apply Dirichlet boundary conditions, i.e., $\left.G_{D}\left(\mathbf{x}, \mathbf{x}^{\prime}\right)\right|_{z^{\prime}=0}=0$, where $G_{D}\left(\mathbf{x}, \mathbf{x}^{\prime}\right)$ denotes the Green's function for Dirichlet conditions, and the primed vector $\mathbf{x}^{\prime}$ locates the charge distribution points. Using Green's theorem and the fact that the volume

charge density $\rho\left(\mathbf{x}^{\prime}\right)=0$, the electrostatic potential for $z>0$ can be expressed in terms of the value of the potential on the plate $\Phi\left(\mathbf{x}^{\prime}\right)=V$ [11]

$$
\Phi(\mathbf{x})=-\frac{1}{4 \pi} \int \Phi\left(\mathbf{x}^{\prime}\right)\left(\frac{\partial G_{D}}{\partial n^{\prime}}\right) \mathrm{da}^{\prime}
$$


where $\hat{\mathbf{n}}^{\prime}=-\hat{\mathbf{z}}^{\prime}$ is the outward unit normal and $\mathrm{da}^{\prime}$ is an infinitesimal area element of the plate.

By employing the method of images [11], we can easily find the Green's function by considering the potential in $\mathbf{x}$ due to a unit point charge located at a point in the region $z>0$, plus the potential of an image charge placed in a symmetric position in the lower-half plane $z<0$. Through this procedure we calculate $\left(\partial G_{D} / \partial n^{\prime}\right)=\left.\left(-\partial G_{D} / \partial z^{\prime}\right)\right|_{z^{\prime}=0}$ explicitly, and rewrite equation (4) as

$$
\Phi(\mathbf{x})=\frac{V}{2 \pi} \Omega(\mathbf{x})
$$

where

$$
\Omega(\mathbf{x})=\int \frac{(-\hat{\mathbf{z}}) \cdot\left(\mathbf{x}^{\prime}-\mathbf{x}\right)}{\left|\mathbf{x}-\mathbf{x}^{\prime}\right|^{3}} \mathrm{da}^{\prime}
$$

is the solid angle subtended by the surface (flat plate of area $a^{\prime}$ ) spanning the loop $C$ as seen from $\mathbf{x}$. The sign convention established for the solid angle is the following: $\Omega(\mathbf{x})$ is positive when the observation point $P$ views the "inner" side of the flat plate, in other words, $\Omega(\mathbf{x})>0$ if the unit normal points away from $P$. This sign convention is the same as the one adopted in references [3,4t.

By substituting equations (5) and (6) into the electric field equation (3) and using the well-known relation 12

$$
\nabla \Omega(\mathbf{x})=\oint_{C} \frac{\mathbf{d s}^{\prime} \times\left(\mathbf{x}-\mathbf{x}^{\prime}\right)}{\left|\mathbf{x}-\mathbf{x}^{\prime}\right|^{3}}
$$

we finally obtain

$$
\mathbf{E}(\mathbf{x})=\frac{V}{2 \pi} \oint_{C} \frac{\left(\mathbf{x}-\mathbf{x}^{\prime}\right) \times \mathbf{d s}^{\prime}}{\left|\mathbf{x}-\mathbf{x}^{\prime}\right|^{3}}
$$

where $\mathbf{d} \mathbf{s}^{\prime}$ is an element of length of the integration path $C$. The direction of the integration around $C$ is determined by the direction of the outward unit normal via the right-hand 
rule. Notice that the cross-product in (8) has a reversed order in comparison to the one that appears in the magnetostatic case (四). This distinction is necessary to give the correct direction for the electric field vector at the observation point $P$.

Equation (8) is our central result. It allows the calculation of the electric field through a Biot-Savart-type law. Notice that (8) is written in terms of a line integral, so to calculate the

electric field we just need to take into account the contributions coming from the boundary contour $C$. This result makes the solution of the electrostatic problem much easier. Under the circumstances studied in this work, the traditional Dirichlet problem can be mapped onto a Biot-Savart-like problem, a mapping that can simplify considerably the computation of the electric field in many problems of interest.

As it was in the magnetic case [10], a much simpler expression for the field can be obtained if we concentrate our attention in the calculation of the electric field at observation points that lie in the plane of the charge distribution. Using the same arguments as those presented in reference [10 it can be easily shown that

$$
\mathbf{E}(\mathbf{x})=\frac{V}{2 \pi} \oint_{C} \frac{d \theta}{r} \hat{\mathbf{z}}
$$

where $r=\left|\mathbf{x}-\mathbf{x}^{\prime}\right|$. Equation (9) is a surprisingly simple result. Note that this line integral expression (9) works for flat charge distributions of any boundary shape, including those described by curves $C$ which are not single valued functions of $\theta$. In addition, it is valid for observation points $P$ located either inside or outside the loop $C$. To illustrate our approach, in the next section we calculate the electric field due to specific planar charge configurations of some representative shapes.

\section{ILLUSTRATIVE EXAMPLES}

In this section we illustrate the usefuness the the Biot-Savart-like law (8) by discussing a class of electrostatic problem that is trivially solved by using (8) or (9), but that would 
require a much more involved solution otherwise. We start by applying equation (8) to calculate the electric field due to a thin, flat plate, which has the shape of a regular n-sided polygon, inscribed in a circle of radius $a$ (see figure 3). Consider that the flat plate is located in the $x-y$ plane with its center at the origin, and that it is maintained at a fixed potential $V$. In the plane $z=0$ the region outside the plate is held at zero potential. We wish to compute the electric field at a point $P$ in the $z$-axis.

As discussed in section 2, by inspecting equation (\$) we notice that in order to calculate the electric field due to this flat configuration, we just need to take into account the contributions coming from the boundary contour $C$. Let us analyze this point a little more carefully: the total electric field at point $P$ in the $z$-axis can be written as the superposition of the field due to $n$ triangles obtained by joining the center of circle to the vertices of the polygon (figure 3). If we sum all the contributions from these various triangular paths, we will be left with the integration around the contour $C$. This happens because the sense of integration along their common sides is opposite for two adjacent triangles, making the contributions from the common sides to cancel. Therefore, after performimg the integration around all the triangles, the only nonzero contributions come from the $n$ straight edges that define the plate's boundary contour $C$. With these considerations in mind we turn to the field calculation itself.

When the field contributions of the $n$ sides are summed vectorially, the horizontal $(x-y$ plane) components add to zero. By symmetry, only the vertical components located along the $z$-axis will survive. From figure 3 we verify that the total electric field at $P$ is given by

$$
E=n E_{n} \cos \alpha
$$

where $E_{n}$ is the net field due to just one of the $n$ triangles that compose the polygon and $\cos \alpha=a \cos (\pi / n) / \sqrt{a^{2} \cos ^{2}(\pi / n)+z^{2}}$. Considering the fact that the only nonzero contribution from such triangular path comes from the single edge of the polygon, we employ 
equation (8) to get

$$
E_{n}=\frac{V}{\pi} \frac{a \sin (\pi / n)}{\sqrt{\left[a^{2} \cos ^{2}(\pi / n)+z^{2}\right]\left[a^{2}+z^{2}\right]}} .
$$

Using equations (10) and (11) we obtain the total electric field along the $z$-axis

$$
\mathbf{E}=\frac{V}{2 \pi}\left\{\frac{n a^{2} \sin (2 \pi / n)}{\left[a^{2} \cos ^{2}(\pi / n)+z^{2}\right] \sqrt{a^{2}+z^{2}}}\right\} \hat{\mathbf{z}} .
$$

This result is quite handy since it provides, all at once, the calculation of the electric field along the $z$-axis due to any regular polygon of $n$ sides (equilateral triangle, square, pentagonal, etc.). We point out that our electric field result (12) agrees with the equivalent (but conceptually distinct) formula for the magnetic field at the axis of an n-sized polygonal, current carrying circuit, previously obtained in reference [13].

It is worth mentioning that result $(\sqrt{12})$ can also be used compute the magnitude of the electric field along the axis passing through the center of a circular conducting plate of radius a. This can be easily obtained by taking the limit $n \rightarrow \infty$ in equation (12) yielding

$$
\mathbf{E}=\frac{V a^{2}}{\left[a^{2}+z^{2}\right]^{3 / 2}} \hat{\mathbf{z}}
$$

This limit agrees with reference [14], in which the potential calculation is done solely for the circular plate, by employing traditional boundary-value problem techniques.

We conclude this section by calling the attention of the reader to the fact that the calculation of the electric field at observation points lying in the plane of flat plates of various shapes can be obtained, with great facility, by directly using our expression (9). For example, the magnitude of the electric field in the plane of elliptical, spiral shaped and harmonically deformed circular plates (see figure 4), kept at a fixed potential $V$, are readily obtained by (9). Table 1 displays the values of the electric field at observation points $P$, for these three characteristic flat regions. So, despite the boundary curve especific geometry, the $E$ field calculation may be promptly performed by using our Biot-Savart-like approach. 
In practical terms the difficulty of having a complicated boundary shape is not a very serious obstacle in order to compute $E$ in closed form, as long as the related integrations are not terribly hard to handle.

\section{CONCLUDING REMARKS}

In this work we show that, under certain circumstances, complicated electrostatic problems may have straightforward solutions, if solved by a Biot-Savart-like approach. We consider the general situation in which a flat region of arbitrary shape is kept at a fixed potential $V$, in a otherwise grounded plane. The objective is to compute the electric field due to this nontrivial charge distribution at a given point in space. At first glance, such a calculation looks very involved, mainly because we have no prior knowledge about the precise charge distribution on the planar region. We derived a Biot-Savart-like law suitable to deal with such electrostatic situation, allowing the calculation of the electric field to be done in a simple fashion.

This Biot-Savart-like law is written in terms of a line integral along the boundary contour defined by the nonzero potential region, so that the calculation does not require information about the charge distribution in the bulk. As a by-product, we show that for observation points located in the $z=0$ plane, the Biot-Savart-like expression can be written in a very simple and compact form, as was the case for similar magnetic field calculations recently published in reference [10]. We illustrate our results by calculating the electric field along the axis of a polygonal flat region of $n$ sides, kept at a constant potential $V$. In addition, we calculate the magnitude of the electric field in the plane of charged plates presenting nontrivial boundary geometries such as elliptical, spiral and regularly undulating borders. In summary, we show that it is possible to define a Biot-Savart-like in electrostatics. Our new approach provides an alternative and simple technique to solve a class of complicated 
boundary value problems in electrostatics.

\section{ACKNOWLEDGMENTS}

This work was supported by CNPq and FINEP (Brazilian Agencies). 


\section{REFERENCES}

[1] Halliday D, Resnick R and Walker J 1997 Fundamentals of Physics (New York: John Wiley \& Sons) pp 729-732

[2] Griffiths D J 1989 Introduction to Electrodynamics (New Jersey: Prentice Hall) pp 207211

[3] Jackson J D 1975 Classical Electrodynamics (New York: John Wiley \& Sons) pp 169-173

[4] Eyges L 1980 The Classical Electromagnetic Field (New York: Dover Publications, Inc.) pp $117-123$

[5] Kroener E 1955 Dislocations and the Biot-Savart Law Proc. Phys. Soc. London A 68 53-55

[6] Jackson D P, Godstein R E and Cebers A O 1994 Hydrodynamics of Fingering Instabilities in Dipolar Fluids Phys. Rev. E 50 298-307

[7] Kessler D A and Levine H 1991 Maximal Dendrite Size in Monolayer Systems Phys. Rev. Lett. 67 3121-3123

[8] Goldstein R E, Jackson D J and Dorsey A T 1996 Current-loop Model for the Intermediate State of Type-I Superconductors Phys. Rev. Lett. 76 3818-3821

[9] Buck G 1998 Most Smooth Closed Space Curves Contain Approximate Solutions of the n-Body Problem Nature 395 51-53

[10] Miranda J A 2000 Magnetic Field Calculation for Arbitrarily Shaped Planar Wires Am. J. Phys. 68 254-258

[11] See reference [3], pp 42-45 and reference [4], pp 82-87

[12] See, for instance, reference [4], p 122 
[13] Chirgwin H B, Plumpton C and Kilmister C W 1972 Elementary Electromagnetic Theory, Volume 2, (New York: Pergamon Press) p 215, problem 3

[14] See reference [3], p 79, problem 2.3

[15] Gradshteyn I S and Ryzhik I M 1994 Table of Integrals, Series, and Products (New York: Academic Press) pp 907-913 


\section{Figure Captions}

Figure 1: Two equivalent systems: (a) arbitrarily shaped, planar wire carryng a steady current $I$, and (b) a flat charge distribution of the same shape, maintained at a fixed potential $V$, with zero potential in the rest of the plane. In (a) the magnetic field $\mathbf{B}$ can be calculated

with the help of the usual Biot-Savart law (equation (1)), while in (b) the calculation of the electric field $\mathbf{E}$ may be performed with the help of an electrostatic Biot-Savart-like law (equation (8)).

Figure 2: Charged flat plate located in the $x-y$ plane, bounded by the curve $C$ and kept at a fixed potential $V$. The potential is set to zero in the region of the plane $z=0$ outside the curve $C$. Vector $\mathbf{x}^{\prime}$ locates the source point, while vector $\mathbf{x}$ refers to the field point. The observation point is denoted by $P$.

Figure 3: Schematic view of a regular $n$-sized polygonal plate, kept at a potential $V$. The plate lies in the $x-y$ plane, while the observation point $P$ is located along the $z$-axis. Each individual triangle defines the angle $2 \pi / n$. The direction of integration around $C$ is determined by the outward unit-normal $\hat{\mathbf{n}}=-\hat{\mathbf{z}}$ via the right-hand rule.

Figure 4:(a) Elliptical plate, presenting major axis $2 a$ and minor axis $2 b$, centered at point P; (b) Harmonically deformed plate (Perturbed Circle) $r(\theta)=a[1+\epsilon \cos (n \theta)]$, centered at $P$, for $n=5$ and $\epsilon=0.5$; (c) Logarithmic spiral plate. The magnitude of the electric fields at observation point $P$ due to these shapes are listed in Table 1. 


\section{TABLES}

\begin{tabular}{|c|r|r|}
\hline \hline Boundary Shape & Parametric Equation & Electric Field Magnitude \\
\hline \hline Elliptical & $r(\theta)=\frac{a b}{\sqrt{a^{2} \sin ^{2} \theta+b^{2} \cos ^{2} \theta}}$ & $E=\frac{2 V}{\pi a} \mathcal{G}\left(\sqrt{1-\frac{a^{2}}{b^{2}}}\right)$ \\
\hline \hline Perturbed Circle & $r(\theta)=a[1+\epsilon \cos (n \theta)]$ & $E=\frac{V}{a \sqrt{1-\epsilon^{2}}}$ \\
\hline \hline Spiral & $r(\theta)=q e^{p \theta}$ & $E=\frac{V}{2 \pi q}\left[\frac{1-e^{-2 \pi p}}{p}\right]$ \\
\hline \hline
\end{tabular}

TABLE I. Electric field in the plane of flat regions presenting nontrivial boundary shapes.

Note: the function $\mathcal{G}$ denotes the complete elliptic integral of the second kind [15]. 


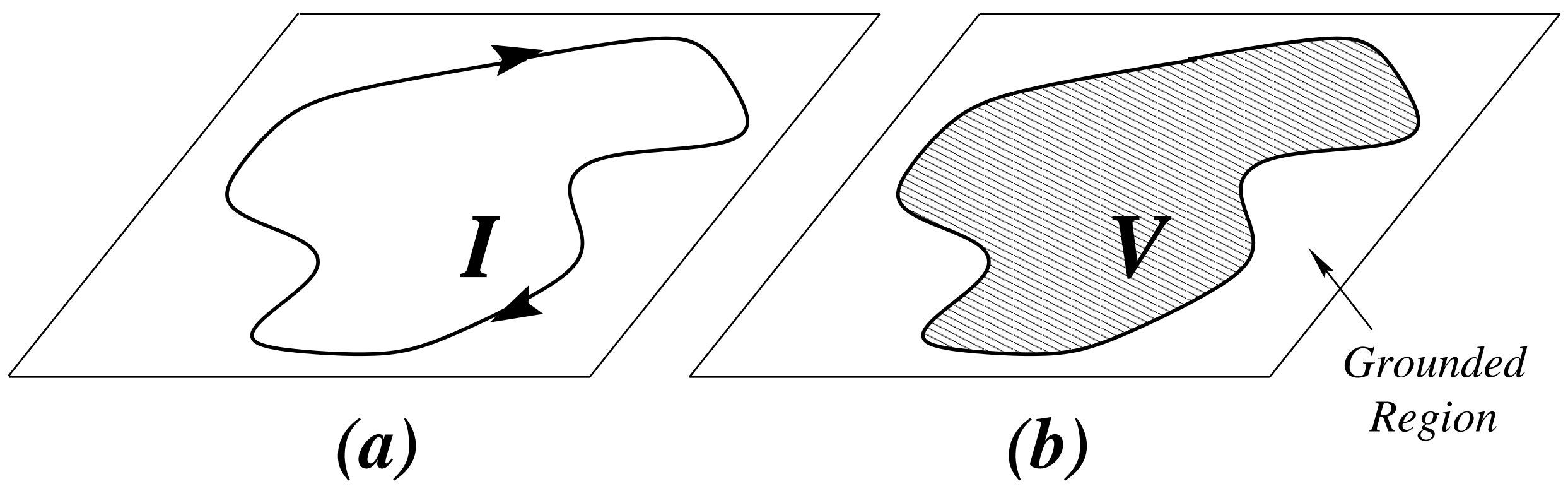




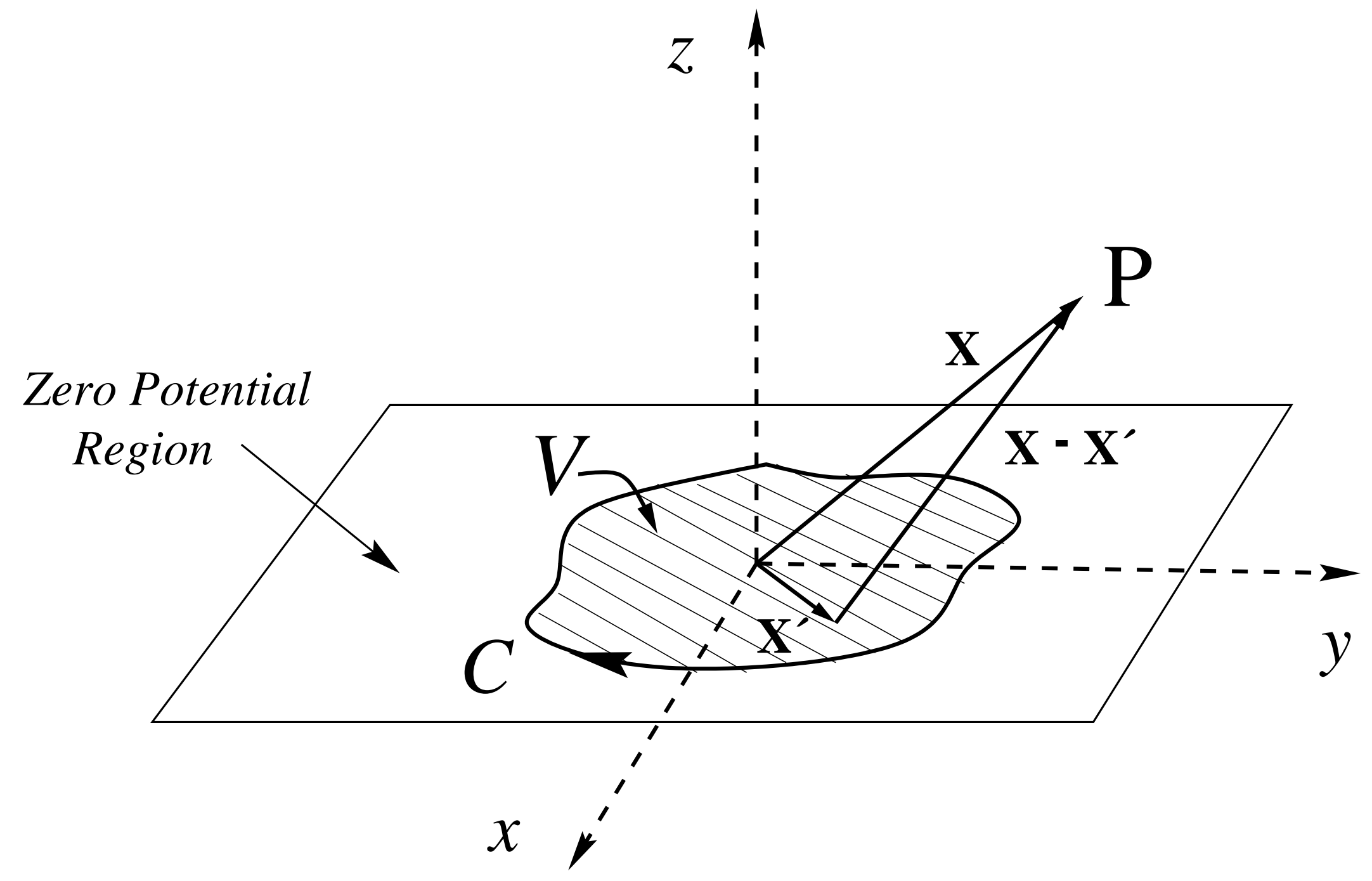




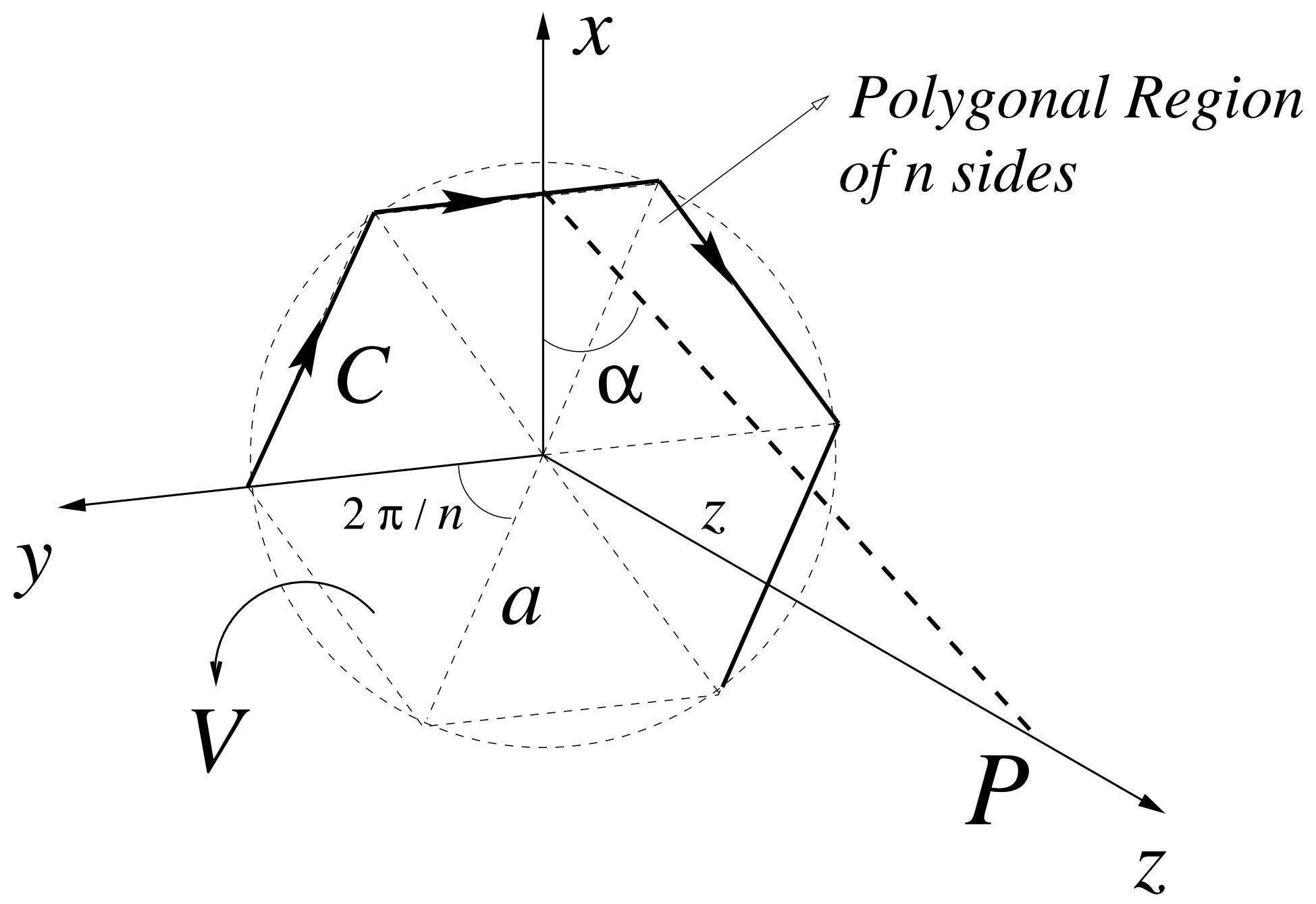


(a)

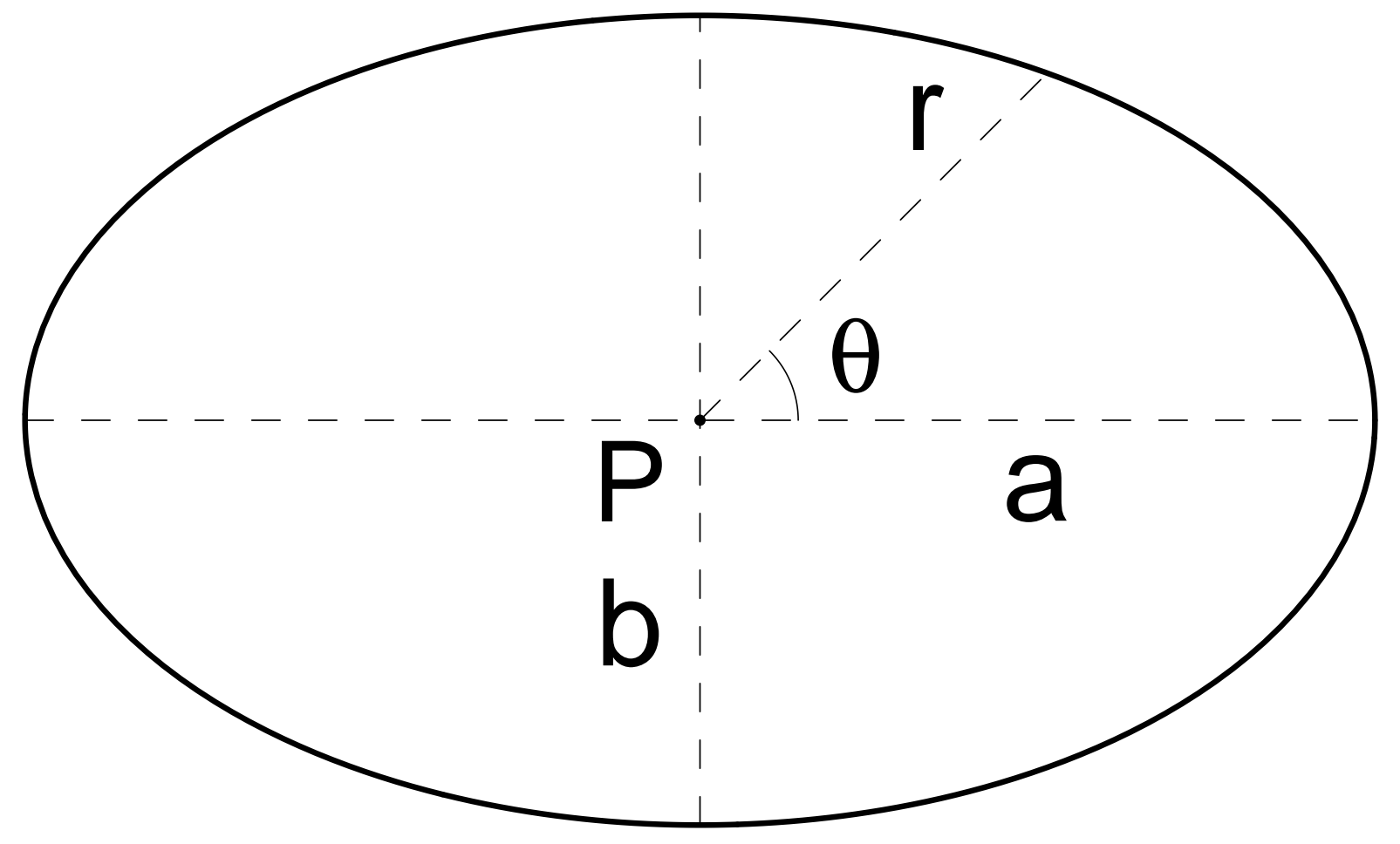




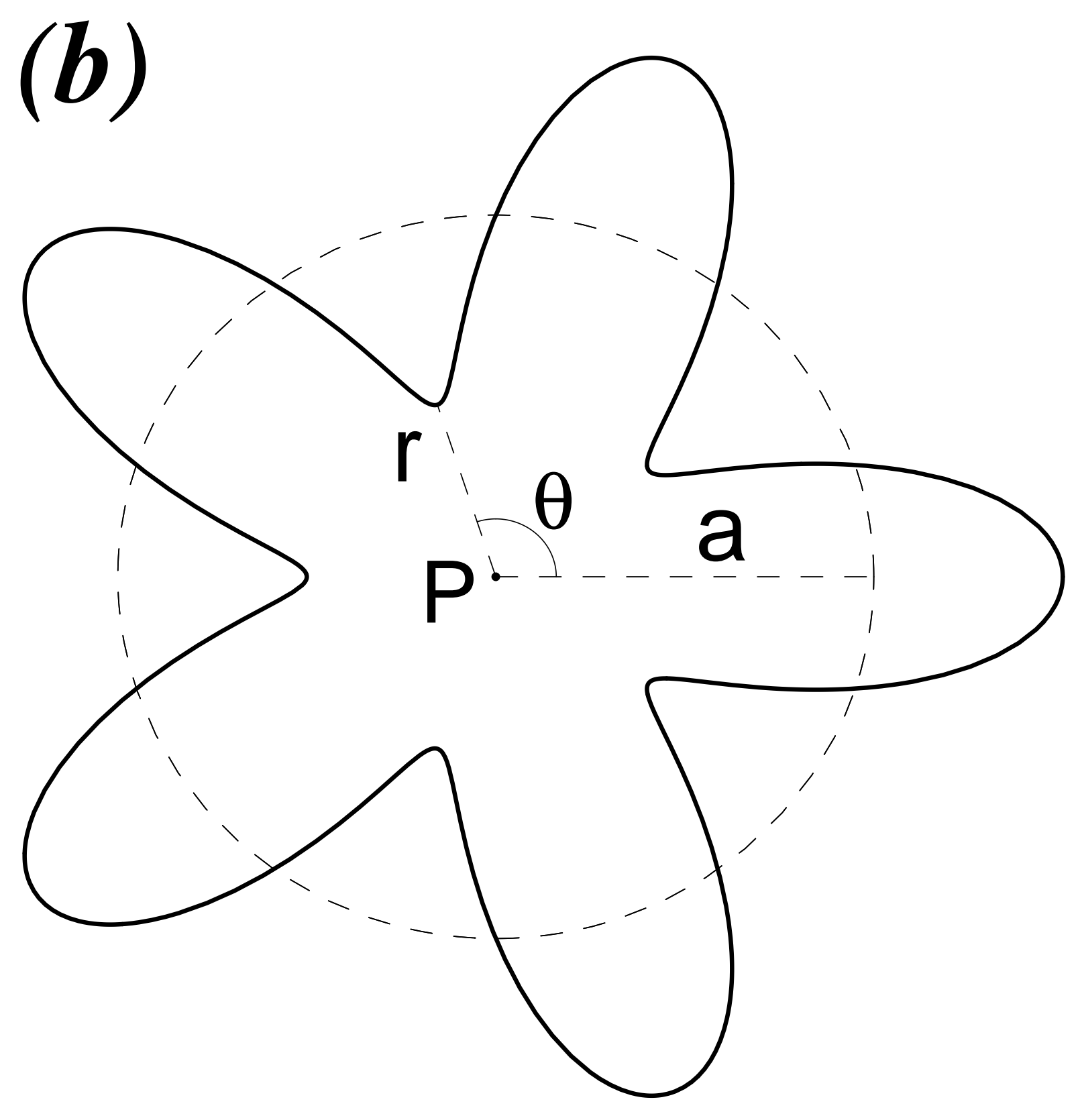




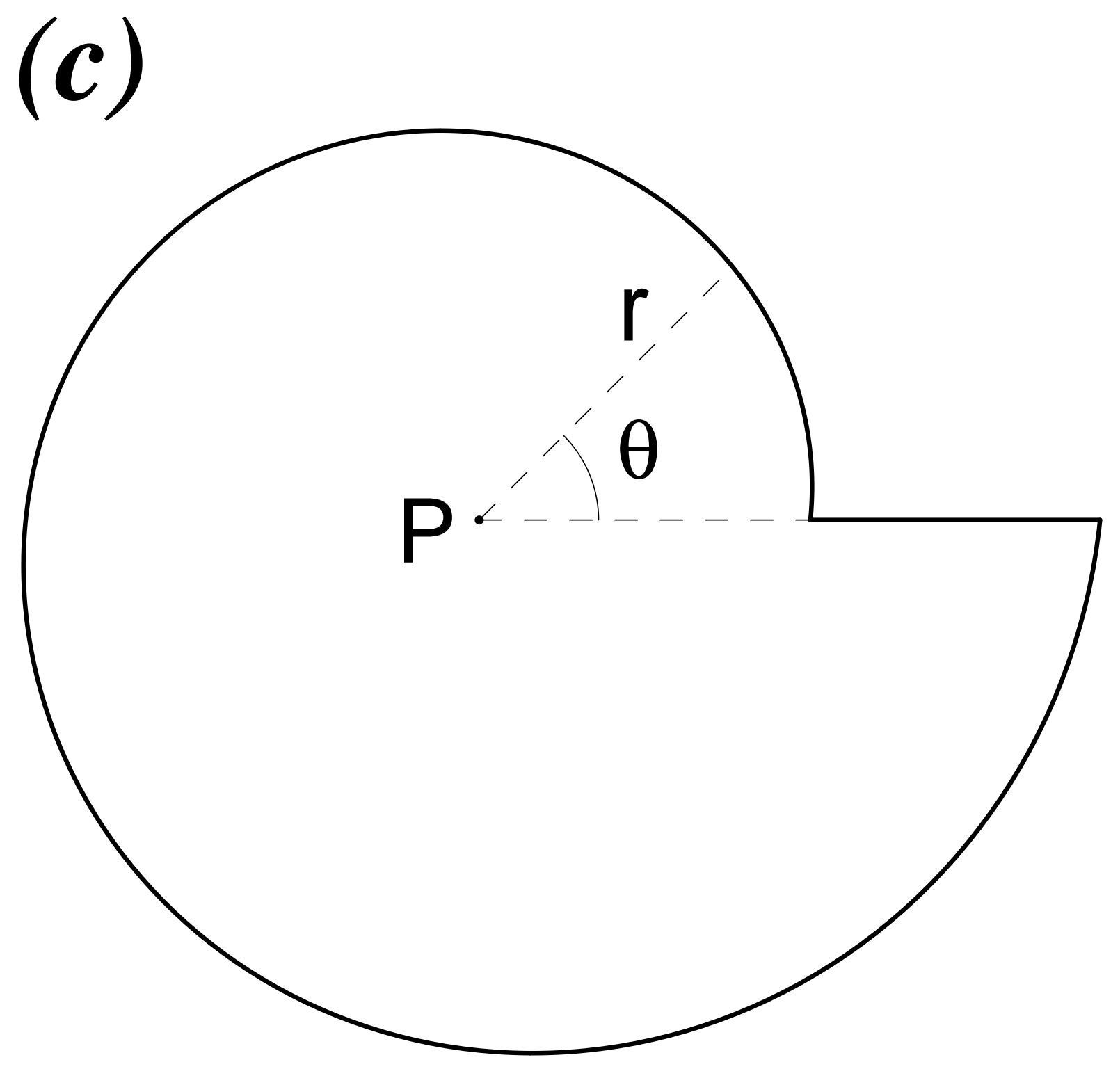

